\title{
Unusual Adsorption at the Air-Water Interface of a Zwitterionic Carboxybetaine with Large \\ Charge Separation
}

\author{
Kun Ma, ${ }^{\dagger}$ Pei Xun Li, ${ }^{\dagger}$ Chu Chuan Dong, ${ }^{\dagger}$ Robert K.Thomas, ${ }^{* \dagger}$ and Jeffrey \\ Penfold \\ $\dagger$ Physical and Theoretical Chemistry Laboratory, South Parks Road, Oxford, OX1 3QZ, UK. \\ $\ddagger$ Rutherford-Appleton Laboratory, Chilton, Didcot, Oxon \\ \Physical and Theoretical Chemistry Laboratory, South Parks Road, Oxford, OX1 3QZ, \\ $U K$. \\ E-mail: robert.thomas@chem.ox.ac.uk
}

\begin{abstract}
The structures of layers of three different dodecylcarboxybetaine surfactants adsorbed at the air-water interface have been determined by neutron reflection. The zwitterionic compounds differed in the length of the spacer separating the quaternary ammonium and carboxylate groups, which was $\left(\mathrm{CH}_{2}\right)_{1},\left(\mathrm{CH}_{2}\right)_{4}$ or $\left(\mathrm{CH}_{2}\right)_{8}$. The limiting areas per molecule were found to be respectively 45, 52 and $84 \AA^{2}$ and compared reasonably with results from surface tension showing that the Gibbs prefactor is 1 in each case. Isotopic labelling was used to distinguish between the position of the alkyl and spacer groups in the layer. The spacer was found to be well immersed in water for the $\left(\mathrm{CH}_{2}\right)_{1}$ and $\left(\mathrm{CH}_{2}\right)_{4}$ spacers but significantly above water for the $\left(\mathrm{CH}_{2}\right)_{8}$ spacer. The distribution of the $\left(\mathrm{CH}_{2}\right)_{8}$ spacer along the surface normal was found to be similar to that of the dodecyl group, i.e. it projects out of the water, contrary to an earlier
\end{abstract}


hypothesis that it forms a loop. Comparison of the overlap of water with dodecyl and spacer groups also indicates that the $\left(\mathrm{CH}_{2}\right)_{8}$ spacer is well out of the water. This in turn suggests that the anionic carboxylic acid group, which is dissociated in solution, is not ionized in the adsorbed layer. A further observation is that the dodecylcarboxybetaine with the $\left(\mathrm{CH}_{2}\right)_{8}$ spacer reaches surface saturation at one tenth of the CMC. This is highly unusual and is attributed to the long spacer destabilizing the micelle relative to the surface layer.

Keywords: Air-water interface, surface structure, surfactant structure, zwitterionic surfactant, neutron reflection, surfactant fragment distribution

\section{Introduction}

The head groups of zwitterionic surfactants contain a pair of opposite charges separated by a spacer. If the spacer is not too large the surfactant will generally carry no nett charge at the natural $\mathrm{pH}$ and adsorption at the air-water interface is then governed by the Gibbs equation. For a 1:1 ionic surfactant this is

$$
\frac{d \gamma}{R T}=-\Gamma_{s} d \ln a_{s}+\Gamma_{i} d \ln a_{i} \approx 2 \Gamma_{s} d \ln c_{s}
$$

where $\gamma$ is the surface tension, $\Gamma$ is the surface excess defined relative to a surface plane for which $\Gamma_{\text {water }}$ is zero, $a$ is the activity and is usually approximated as concentration $c$, and the subscripts $s$ and $i$ indicate surfactant ion and any other ions that are also adsorbed. If the only ion adsorbed apart from the surfactant is the surfactant counterion then neutrality requires the two surface excesses and the two concentrations to be identical and the equation simplifies as shown on the right. For a zwitterionic where both groups are ionized there are no additional ions adsorbed and the Gibbs prefactor in the expression on the right is therefore 1 but if only one of the zwitterionic groups is ionized then surface neutrality requires the adsorption of a counterion and, unless the concentration of this counterion is maintained constant, the Gibbs prefactor then becomes 2, as for a 1:1 ionic surfactant. The 
strength of adsorption and the critical micelle concentration (CMC) tend to be intermediate between those of nonionic and ionic surfactants and the presence of the two charges makes zwitterionics very soluble e.g. ${ }^{1}$ These properties and the lack of overall charge, which weakens the interaction with species such as proteins, confers a mildness on zwitterionic surfactants that is useful for biological applications ${ }^{2}$ and in formulations for personal care. ${ }^{3}$

The length of the spacer between the charges in a zwitterionic has a strong effect on the interaction between the two charges especially if one of them is weak. ${ }^{4}$ In a carboxybetaine (see Figure 1) the quaternary ammonium is a permanent charge but the carboxyl group is a weak acid group, which would have a $\mathrm{pK}_{a}$ typically in the range $4-5$ if simply connected to an alkyl group. However, when the carboxyl group is separated from a cationic group by only one methylene group the $\mathrm{pK}_{a}$ drops substantially and the zwitterionic is therefore fully charged at natural $\mathrm{pH}$. For example, in the $\alpha, \omega$-ammonioalkanoates the change in $\mathrm{pK}_{a}$ is from 2.3 for a single methylene spacer to 4.5 for a hexamethylene spacer, which is close to the value of 4.7 for acetic acid. ${ }^{5}$ In the ammonioalkanoates both charged groups are weak electrolytes and the behaviour might be slightly different when the cationic group is a quaternary alkylammonium group. However, in dodecylcarboxybetaine with a single methylene spacer the $\mathrm{pK}_{a}$ is $2.4^{6}$ and rises similarly to the ammonioalkanoates as the spacer length increases. ${ }^{7}$ Thus the added stabilization of the dissociated state is gradually lost as the length of the spacer is progressively increased. The effect has been most thoroughly examined for the ionization of dibasic carboxylic acids where the second dissociation is strongly affected by the presence of the negative charge from the first ionization and the effect should be similar but opposite in sign in a zwitterionic. These studies have shown that there are several subtleties concerning the local dielectric environment, ${ }^{8-12}$ and these may also be important at the surface and in micelles, but the general result that the $\mathrm{pK}_{a}$ of the carboxylic acid group in the zwitterionic should increase by about 2 units when the spacer changes from 1 to more than about 6 methylene units is a reliable one. A complication at the surface is that an undissociated carboxylic acid part of the spacer/head group will 
be less hydrophilic/more hydrophobic and this may contribute in a different way to the surface properties. In addition to the possible variation of charge with chainlength the dipole associated with the charge also varies linearly with the separation in a rigid spacer but more slowly in the typical flexible alkyl spacer of the zwitterionics that have so far been studied.

Chevalier et al. have investigated the effect of the separation of the charges on the surface behaviour of two series of zwitterionics, phosphocholines and dodecylcarboxybetaines, where the spacer was varied from $\mathrm{CH}_{2}$ to $\left(\mathrm{CH}_{2}\right)_{10} \cdot{ }^{13,14}$ In both series the critical micelle concentration (CMC) passes through a pronounced maximum at around $n=4$ where $n$ is the number of methylene units and this was explained in terms of the repulsion between the zwitterionic dipoles at the micellar surface as a function of $n$ (Chevalier et al. determined the actual separation of the charges using NMR measurements on the isolated molecules). Chevalier et al. made a more complete study of a second series of dodecylcarboxybetaines. In this series the limiting area per molecule at the critical micelle concentration (CMC), $A_{C M C}$, increases steadily with spacer length from 52 at a spacer length of 1 carbon to to 92 $\AA^{2}$ at 10 carbons, but the CMC and the limiting surface tension at the CMC, $\gamma_{C M C}$, both show strong maxima in the region of 3-4 methylene groups. ${ }^{14}$ The $\mathrm{CMC}$ was explained as for the phosphocholine series but the more steady increase of $A_{C M C}$ was interpreted in terms of a balance of the dipolar repulsion and an increasing steric lateral repulsion with spacer length which eventually causes the more flexible long spacers to form a loop at the surface. Given that the properties of these important surfactants can evidently be fine tuned by adjustment of the spacer length, the understanding of the surface and aggregation behaviour as a function of spacer length is important, and this is best done using a technique that can explore the surface and/or aggregate structure directly.

Neutron reflection in conjunction with deuterium labelling offers a means for studying the distributions of surfactant fragments along the direction normal to the surface ${ }^{15,16}$ and should be able to identify structural changes associated with changes of the spacer orientation or 
position relative to the underlying water. In addition, the combination of neutron reflection with surface tension measurements allows an accurate assessment of the application of the Gibbs equation to the surface tension data, which may also reveal interesting features of the surface behaviour, e.g. ${ }^{17,18}$ For example, in the surface tension data of Chevalier et al. there is an unusually long linear stretch of the ST curve for the largest of the spacers studied but this was not commented on in the original paper. We have now measured neutron reflection from the surfaces of the deuterated species, using differently labelled molecules to separate the signals from the chain and spacer moities, and also repeated the surface tension measurements of Chevalier et al. for a selection of the same series of dodecylcarboxybetaines with spacers of 1, 4 and 8 methylene groups (Figure 1).

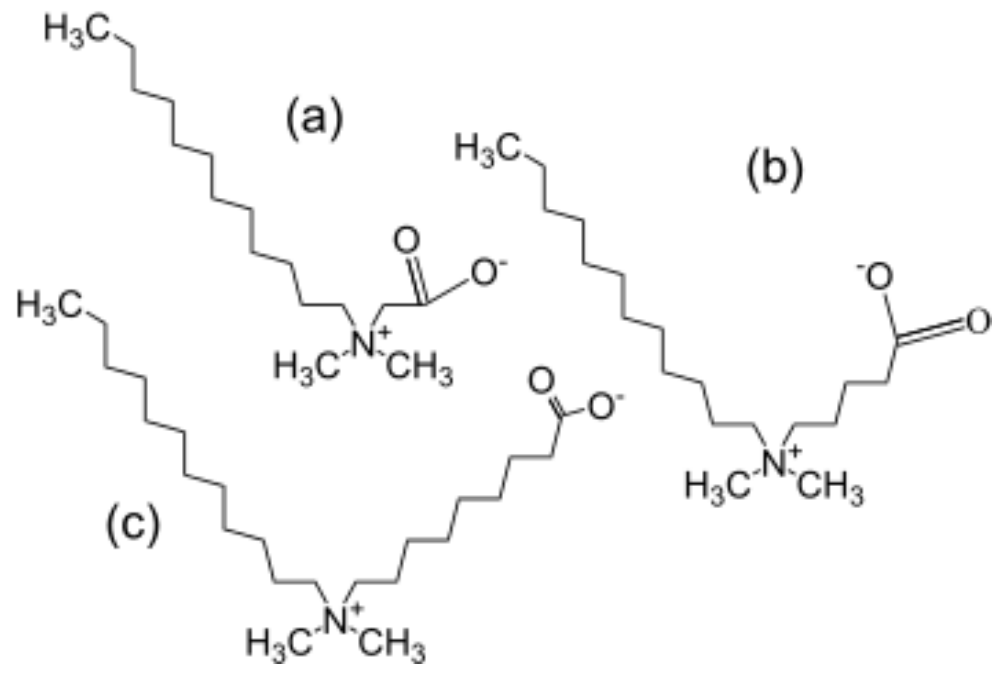

Figure 1: Fully extended conformations of dodecylcarboxy betaine with (a) 1, (b) 4 and (c) 8 methylene long spacers $\left(\mathrm{C}_{12} \mathrm{C}_{n}\right.$ betaine). The carboxylate has been drawn as the fully ionized form which dominates in dilute solution, even for the most weakly acidic group in $\mathrm{C}_{12} \mathrm{C}_{8}$ betaine. The $p K_{a}$ values are (a) $2.4^{6}$ and (b) 4.3 and (c) 4.6 (both estimated from dibasic carboxylic acid data. ${ }^{8}$

\section{Experimental Details}

The three dodecylcarboxybetaine surfactants with $\mathrm{C}_{1}, \mathrm{C}_{4}$ and $\mathrm{C}_{8}$ spacers between the quaternary ammonium and carboxy groups (designated $\mathrm{C}_{12} \mathrm{C}_{n}$ betaine in the rest of the paper) 
were synthesized following the two step procedure of Weers et al. ${ }^{7}$ Dodecyldimethylamine was reacted with the appropriate bromoalkyl mono ester to form the quaternary ammonium salt with the alkyl monoester using ethyl acetate, 2-propanol or ethanol as solvent depending on the alkyl group in the monoester. The crude ammonium salt was then dissolved in UHQ water and treated with an ion exchange resin. The hydrogen ion associated with the cationic resin promotes base-catalysed ester hydrolysis which results in the liberation of the betaine. The final compounds were recrystallized from an acetone-methanol mixture and obtained as white crystals which were freeze-dried. The purity was assessed by the absence of a minimum in the plot of surface tension against $\ln c$. The chain deuterated surfactant was synthesised in the same way as the protonated one by using chain deuterated dodecyldimethylamine.

In order to highlight the neutron reflectivity signal from the head-group, $\mathrm{C}_{12} \mathrm{C}_{4}$ betaine and $\mathrm{C}_{12} \mathrm{C}_{8}$ betaine were also synthesised with their head-group deuterated. For the $\mathrm{C}_{4}$ spacer the deuterated 5-bromovalerate used in the procedure above was made using the reaction scheme

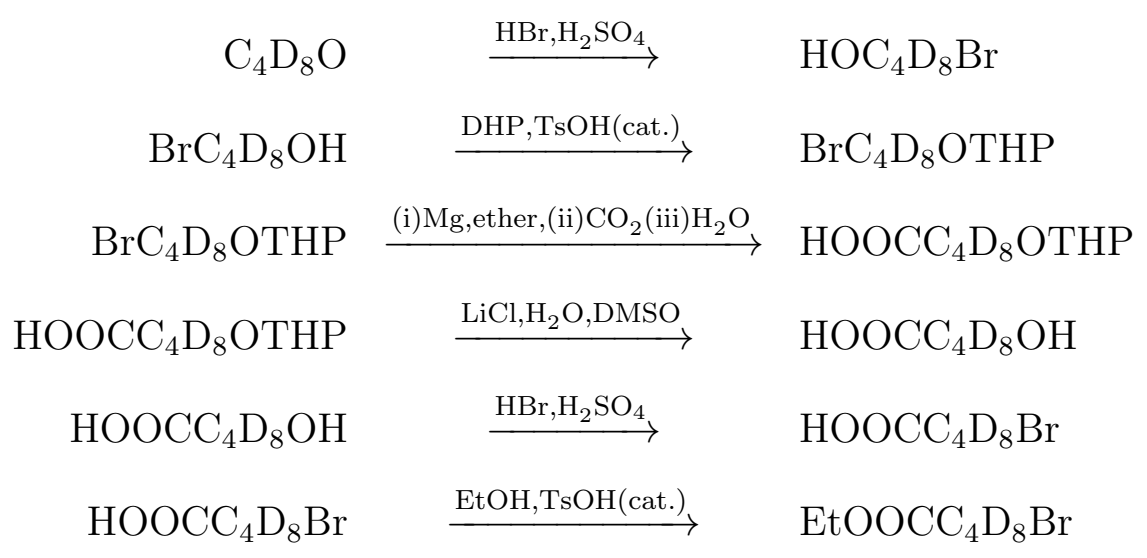

starting from perdeuterated THF. Concentrated $\mathrm{H}_{2} \mathrm{SO}_{4}(3 \mathrm{ml})$ and then $48 \mathrm{ml} \mathrm{HBr}(48 \% \mathrm{w} / \mathrm{v}$, $0.42 \mathrm{~mol})$ were added dropwise to $30 \mathrm{~g}$ deuterated THF $(0.4 \mathrm{~mol})$ at $0{ }^{\circ} \mathrm{C} .{ }^{19,20}$ The mixture was stirred and refluxed for 90 minutes and, after cooling to $0^{\circ} \mathrm{C}$, was neutralized with $\mathrm{NaHCO}_{3}$ and $50 \mathrm{ml}$ of UHQ water added. The 4-bromo-1-butanol product was extracted with diethyl ether, washed with brine and dried with magnesium sulfate. The yield after removal 
of solvent was $86 \%$. The 2-tetrahydropyranyl group was then introduced as a protecting group for the alcohol group. ${ }^{21} 30 \mathrm{ml}$ dihydropyran $(0.34 \mathrm{~mol})$ was added via a syringe to a cooled solution $\left(0^{\circ} \mathrm{C}\right)$ of 4-bromo-1-butanol $(40 \mathrm{~g}, 0.26 \mathrm{~mol})$ in $100 \mathrm{ml}$ anhydrous ether containing $100 \mathrm{mg}$ of p-toluenesulfonic acid. After an hour at ambient temperature, the reaction mixture was twice washed with saturated sodium bicarbonate solution and then with brine. The organic layer was dried over anhydrous potassium carbonate and filtered. The crude 4-bromobutyl tetrahydropyranyl ether, obtained after removal of solvent, was purified by liquid chromatography with a petroleum ether/ether mixture. The bromo group was replaced by carboxylate using a Grignard reaction. $4.18 \mathrm{ml}(20 \mathrm{~g})$ of 4-bromobutyl tetrahydropyranyl ether and $50 \mathrm{ml}$ of anhydrous THF were added dropwise to 4-bromobutyl tetrahydropyranyl ether/THF solution in a round-bottomed flask to maintain a steady reflux. The freshly made Grignard reagent was poured into excess dry ice for carboxylation of the THP-protected compound. The reaction mixture was then warmed slowly to ambient temperature and extracted with water $(100 \mathrm{ml})$. The aqueous solution was washed with diethyl ether and the extracts dried and concentrated under reduced pressure. The THP ether was deprotected following Maiti et al. ${ }^{22}$ A stirred mixture of $20 \mathrm{~g}$ THP ether $(0.1 \mathrm{~mol})$, $20 \mathrm{~g} \mathrm{LiCl}(0.5 \mathrm{~mol})$, and $18 \mathrm{~mL} \mathrm{H} \mathrm{O}^{\mathrm{O}}(1 \mathrm{~mol})$ in DMSO $(100 \mathrm{ml})$ was heated at $90^{\circ} \mathrm{C}$ for 6 hours under $\mathrm{N}_{2}$ protection. ${ }^{23}$ The reaction mixture was cooled to room temperature, diluted with $\mathrm{H}_{2} \mathrm{O}(100 \mathrm{ml})$, and extracted with ether $(100 \mathrm{ml} \times 3)$. The crude hydroxyvaleric acid was dried over anhydrous sodium sulfate and purified on a silica gel column using ethyl acetate/light petroleum $\left(60^{\circ} \mathrm{C}\right.$ to $\left.80^{\circ} \mathrm{C}\right)$ with a yield of $81 \%(9.2 \mathrm{~g})$. The acid was brominated following by Buchi. ${ }^{24} 9.2 \mathrm{~g}$ of the acid were added to an ice cold mixture of $40 \mathrm{ml}$ of $40 \% \mathrm{HBr}$ and $10 \mathrm{ml}$ of $\mathrm{H}_{2} \mathrm{SO}_{4}$. After stirring at room temperature overnight and then in a steam bath for 5 hours, the crude 5-bromovaleric acid was extracted with ether and purified on a silica gel column using petroleum ether (yield $=9 \mathrm{~g}, 49 \%$ ). Finally, ethyl 5-bromovalerate was synthesised following Kurata. ${ }^{25}$ A catalytic amount of 4-toluenesulfonic acid was added to a mixture of excess EtOH (40 ml) and 5-bromo-pentanoic acid (9 g, $0.05 \mathrm{~mol})$ and, after 
refluxing at $70-80^{\circ} \mathrm{C}$ overnight, the crude product was rotary evaporated and purified on a silica gel column using petroleum ether (yield $=7.6 \mathrm{~g}, 73 \%$ ). Owing to the difficulty of using NMR to determine the purity of perdeuterated materials the sequence of reactions above was first done with protonated materials (using ${ }^{1} \mathrm{H}$ NMR for analysis) and then repeated exactly for the deuterated system.

Protonated ethyl 9-bromononanoate for the $\mathrm{C}_{8}$ spacer was synthesised from monoethyl decanoate as the starting compound. ${ }^{26}$ In a $500 \mathrm{ml}$ round bottom flask a mixture of $20.4 \mathrm{~g}$ red $\mathrm{HgO}(0.1 \mathrm{~mol}), 43.25 \mathrm{~g}$ monomethyl decanoate $(0.2 \mathrm{~mol})$ and $200 \mathrm{ml}$ of $\mathrm{CCl}_{4}$ was heated under a $50 \mathrm{~cm}$ Vigreux column, with stirring, such that rate of distillation was about one drop per second. After 15 minutes of distillation in this manner, addition was begun of a solution of $30 \mathrm{~g}$ of bromine in $60 \mathrm{ml}$ of $\mathrm{CCl}_{4}$, added over 70 minutes. In the last stages the majority of the bromine distilled over unchanged. When the bromine addition was complete, a further $60 \mathrm{ml} \mathrm{CCl}_{4}$ was added over 40 minutes, while distillation continued as before. Mercury salts were removed from the cooled reaction mixture by filtration with suction through a filter-aid mat. The clear filtrate was extracted with $50 \mathrm{ml}$ of $5 \%$ aqueous $\mathrm{NaOH}$ and the coagulated precipitate which formed was removed from the two phase solution by suction filtration. After the $\mathrm{CCl}_{4}$ phase had been washed with ultrapure water from a UHQ ion exchange purifier, the ethyl 9-bromononanoate was recovered by distillation. The final product was obtained by using liquid chromatography with petroleum ether and ethyl acetate mixture and the structure was confirmed by ${ }^{1} \mathrm{H}-\mathrm{NMR}$. Deuterated methyl 9-bromononanoate (methyl group not deuterated) was prepared in three stages, the last of which was the same bromination step as described above. Decan-dioic acid was successively deuterated in a Parr reactor using $\mathrm{PtO}$ catalyst, $\mathrm{D}_{2} \mathrm{O}$ and $\mathrm{Na}_{2} \mathrm{O}_{2}$ as described previously to give a product containing 98\% D after three exchanges. The diacid was esterified with methanol and the monomethyl decanoate was isolated from the free diacid and diester by distillation. Bromination as described above finally gave methyl 9-bromononanoate, which was used for preparation of the $\mathrm{hC}_{12} \mathrm{dC}_{8}$ betaine, where $\mathrm{h}$ denotes fully protonated and $\mathrm{d}$ fully deuterated. 
The surface tension measurements were performed on a Krüss K10T digital tensiometer by the du Noüy ring method with a platinum/iridium ring. The apparatus was calibrated and corrected following the manufacturer's protocols. All experiments were done at $298 \pm 1 \mathrm{~K}$.

Neutron reflection (NR) measurements were performed on the reflectometers INTER and SURF at the Rutherford-Appleton Laboratory (Didcot, U.K.). The instruments and the procedure for making the measurements have been described fully elsewhere. ${ }^{27,28}$ Measurements were made at an incident angle of $1.5^{\circ}$, which gives a range of momentum transfer, $\kappa(=$ $(4 \pi \sin \theta) / \lambda$, where $\theta$ is the glancing angle of incidence), from 0.03 to $0.35 \AA^{-1}$, and a flat incoherent scattering background was subtracted. The conversion of measured signal to absolute intensity was made by calibration with $\mathrm{D}_{2} \mathrm{O}$. Independent measurements to determine surface excess were made for the chain deuterated and spacer deuterated surfactants in null reflecting water (NRW). Under these circumstances the reflected signal is entirely from the surfactant layer. The surface excess of surfactant was determined by fitting the NR data with a simple slab model using the standard optical matrix method to calculate the reflectivity. ${ }^{29}$ In this model the layer is characterized by its thickness, $\tau$, and scattering length density, $\rho$. Once the values of $\tau$ and $\rho$ are determined they can be converted into the area per molecule at the surface, $A$, using

$$
\rho=\frac{\Sigma b}{A \tau}
$$

where $b$ are the known scattering lengths of the fragments in the layer, which are given in Table 1 of the Supporting Information. A more extensive series of measurements to determine the structure of the layer were made above the CMC and used the NRW measurements above as well as three measurements in $\mathrm{D}_{2} \mathrm{O}$, of the $\mathrm{hC}_{12} \mathrm{hC}_{n}$ betaine, $\mathrm{dC}_{12} \mathrm{hC}_{n}$ betaine and $\mathrm{hC}_{12} \mathrm{dC}_{n}$ betaine. A modification of the partial structure factor method ${ }^{16}$ was used to analyse this data and is described in the discussion below. 


\section{Results and Discussion}

The surface tension measurements for the three dodecylcarboxybetaines are shown in Figure 2 with fits based on a quadratic in $\ln c$ and a Gibbs prefactor of unity. Included for comparison are the results for the $\mathrm{C}_{10}$ spacer reproduced from Chevalier et al. ${ }^{14} \mathrm{~A}$ feature of particular interest is that the $\gamma-\ln c$ plot for our largest spacer, $\mathrm{C}_{8}$, is linear over a very long range of concentration and this was also found by Chevalier et al. for their largest spacer, $\mathrm{C}_{10}$, which is the reason for including this extra data in the Figure. The numerical results are compared with the results from Chevalier et al. in Table 1. Apart from the surface excess for the $\mathrm{C}_{4}$ compound and a constant shift in calibration of the surface tension the results agree within experimental error.

Table 1: Limiting values of CMC, $\gamma_{C M C}, A_{C M C}$ and $\Gamma_{C M C}$ (from application of the Gibbs equation with prefactor of unity) for three dodecylcarboxybetaines. Results from Chevalier et al. are given in brackets for ${ }^{a} \mathrm{C}_{12} \mathrm{C}_{1}$ betaine and $\mathrm{C}_{12} \mathrm{C}_{4}$ betaine and ${ }^{c}$ for $\mathrm{C}_{12} \mathrm{C}_{7}$ betaine ${ }^{14}$ (they did not measure $\mathrm{C}_{12} \mathrm{C}_{8}$ betaine). ${ }^{b}$ Results from Hines et al. ${ }^{30}$ The surface tension measurements were made at $298 \mathrm{~K}$.

\begin{tabular}{|ccccc|}
\hline Components & $\begin{array}{c}\text { CMC } \\
\pm 20 \% \mathrm{mM}\end{array}$ & $\begin{array}{c}\text { Surface tension } \\
\pm 0.5 \mathrm{mN} \mathrm{m}^{-1}\end{array}$ & $\begin{array}{c}A_{C M C} \\
\pm 15 \% \AA^{2}\end{array}$ & $\begin{array}{c}\Gamma_{C M C} \\
\pm 15 \% \mathrm{~mol} / \mathrm{m}^{2}\end{array}$ \\
\hline $\mathrm{C}_{12} \mathrm{C}_{1}$ betaine & $1.9\left(2.0^{a}\right)$ & $39.8\left(36.7^{a}\right)$ & $44\left(52^{a}, 50^{b}\right)$ & 3.8 \\
$\mathrm{C}_{12} \mathrm{C}_{4}$ betaine & $3.3\left(3.4^{a}\right)$ & $45.4\left(44.6^{a}\right)$ & $53\left(68^{a}\right)$ & 3.1 \\
$\mathrm{C}_{12} \mathrm{C}_{8}$ betaine & $1.0\left(1.5^{c}\right)$ & $42.1\left(41.6^{b}\right)$ & $77\left(77^{c}\right)$ & 2.2 \\
\hline
\end{tabular}

The neutron reflectivity profiles of the partially deuterated carboxybetaines were measured over a range of concentrations from below to above the CMC of each component. Apart from $\mathrm{C}_{12} \mathrm{C}_{1}$ betaine, the isotopic compositions measured were $\mathrm{dC}_{12} \mathrm{hC}_{n}$ betaine and $\mathrm{hC}_{12} \mathrm{dC}_{n}$ betaine, each in null reflecting water (NRW), where $\mathrm{d}$ and $\mathrm{h}$ denote the deuterated and protonated part of the surfactant. The data from NRW gives two independent and direct measurements of the surface excess from each of the partially deuterated species because the signal is only from the surfactant layer. The results of fitting a single uniform layer model to the NRW data are given in Table 2. The value of the surface excess, $\Gamma$, at this contrast depends on the product of scattering length density and layer thickness $(\rho \times \tau$ in the Table, 


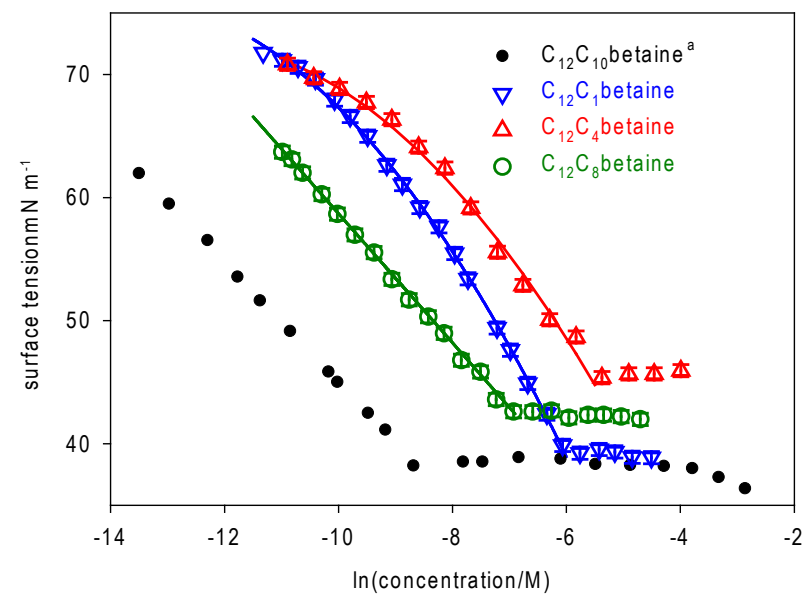

Figure 2: Surface tension measurements for dodecyl carboxybetaines with methylene spacers of 1,4 and 8 units (points) and fits to the Gibbs equation using a quadratic in $\ln c$ and a prefactor of unity. Also included are surface tension data ${ }^{a}$ from Chevalier et al. ${ }^{14}$ for the compound with a spacer of 10 methylene groups. 


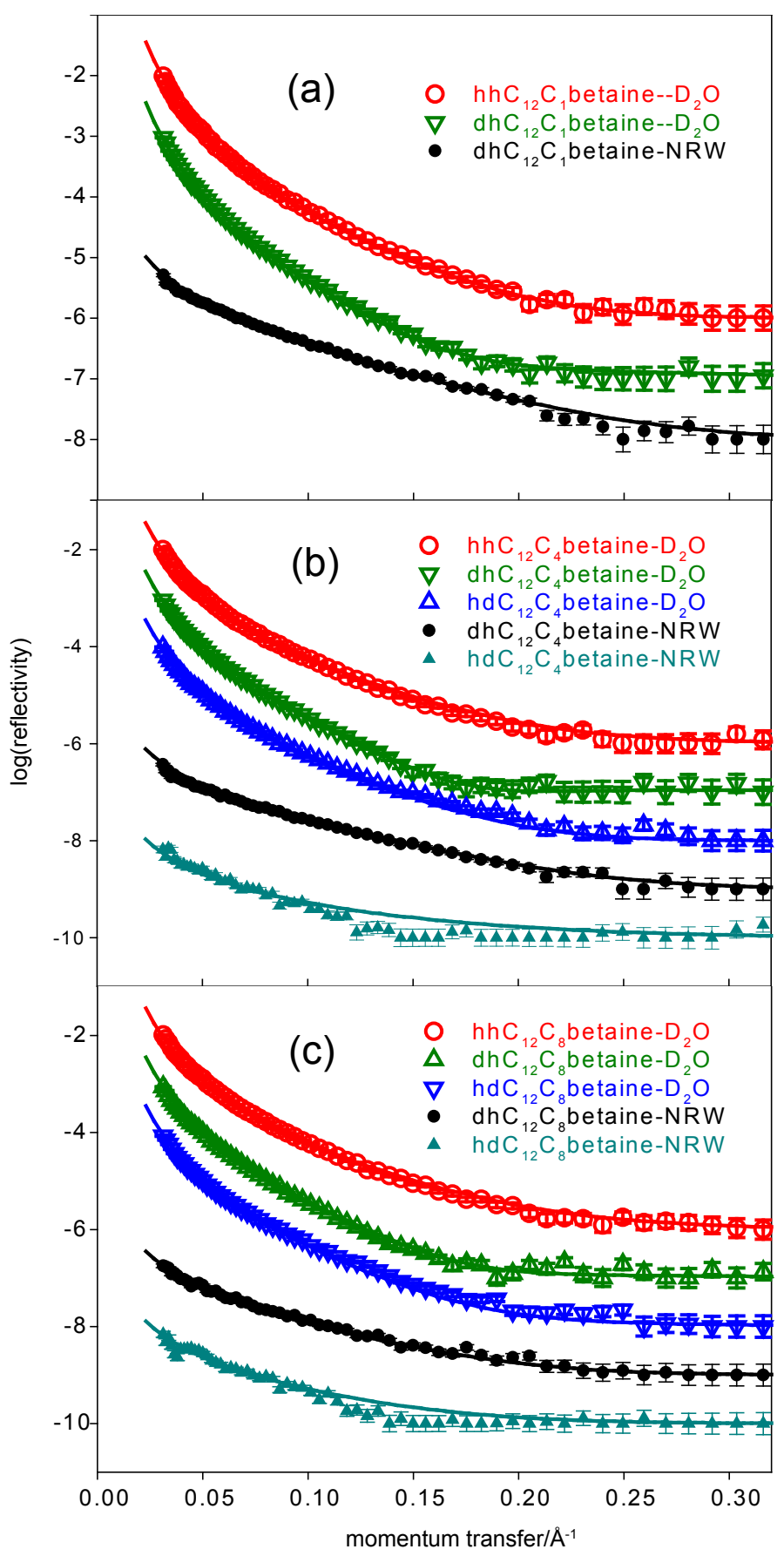

Figure 3: Neutron reflectivity profiles for different isotopic species of dodecyl carboxybetaines with methylene spacers of (a) 1 , (b) 4 and (c) 8 units (points) and the best fits (lines) using the structures shown in Figure 4. For clarity, the individual profiles in each set are successively displaced downwards by 1 unit. 
see also Eqn (2)) and, although individually these vary with a significant error, the product is independent of the model used for the profile of the layer. We do not use these layer thicknesses for a discussion of the layer structure and they are therefore omitted from Table 3. A more extended analysis of the surface structure, using a wider range of measurements, is presented below.

Table 2: Uniform layer fitting results for $\mathrm{C}_{12} \mathrm{C}_{1}$ betaine, $\mathrm{C}_{12} \mathrm{C}_{4}$ betaine and $\mathrm{C}_{12} \mathrm{C}_{8}$ betaine using two different isotopic ompositions of surfactant in null reflecting water at $298 \mathrm{~K}$.

\begin{tabular}{|c|c|c|c|c|}
\hline contrast & $\begin{array}{c}c / \mathrm{mM} \\
\mathrm{mM}\end{array}$ & $\begin{array}{c}\rho \times \tau \\
\pm 5 \% \AA^{-1} \times 10^{6}\end{array}$ & $\begin{array}{c}A \\
\pm 5 \% \AA^{2} \\
\end{array}$ & $\begin{array}{c}\Gamma \\
\pm 5 \% \mu m o l^{-2}\end{array}$ \\
\hline \multirow[t]{6}{*}{$\mathrm{dC}_{12} \mathrm{hC}_{1}$ Betaine } & 0.19 & 32.3 & 80 & 2.1 \\
\hline & 0.56 & 46.8 & 55 & 3.0 \\
\hline & 0.94 & 51.4 & 50 & 3.3 \\
\hline & 1.40 & 55.4 & 47 & 3.5 \\
\hline & 1.87 & 55.4 & 47 & 3.5 \\
\hline & 5.61 & 57.8 & 45 & 3.7 \\
\hline \multirow[t]{6}{*}{$\mathrm{dC}_{12} \mathrm{hC}_{4}$ Betaine } & 0.33 & 32.8 & 78 & 2.1 \\
\hline & 0.99 & 35.6 & 71 & 2.3 \\
\hline & 1.65 & 39.6 & 64 & 2.6 \\
\hline & 2.48 & 43.6 & 58 & 2.9 \\
\hline & 3.30 & 48.4 & 53 & 3.1 \\
\hline & 9.90 & 48.5 & 52 & 3.2 \\
\hline \multirow[t]{6}{*}{$\mathrm{hC}_{12} \mathrm{dC}_{4}$ Betaine } & 0.33 & 8.0 & 82 & 2.0 \\
\hline & 0.99 & 9.6 & 68 & 2.4 \\
\hline & 1.65 & 10.0 & 66 & 2.5 \\
\hline & 2.48 & 11.2 & 58 & 2.9 \\
\hline & 3.30 & 12.5 & 52 & 3.2 \\
\hline & 9.90 & 12.6 & 52 & 3.2 \\
\hline \multirow[t]{6}{*}{$\mathrm{dC}_{12} \mathrm{hC}_{8}$ Betaine } & 0.10 & 29.5 & 85 & 2.0 \\
\hline & 0.31 & 29.0 & 87 & 1.9 \\
\hline & 0.52 & 28.6 & 88 & 1.9 \\
\hline & 0.77 & 30.4 & 83 & 2.0 \\
\hline & 1.03 & 29.4 & 85 & 2.0 \\
\hline & 3.09 & 29.8 & 84 & 2.0 \\
\hline \multirow[t]{6}{*}{$\mathrm{hC}_{12} \mathrm{dC}_{8}$ Betaine } & 0.10 & 17.2 & 86 & 1.9 \\
\hline & 0.31 & 17.5 & 85 & 2.0 \\
\hline & 0.52 & 17.7 & 84 & 2.0 \\
\hline & 0.77 & 17.7 & 84 & 2.0 \\
\hline & 1.03 & 17.5 & 85 & 2.0 \\
\hline & 3.09 & 17.6 & 84 & 2.0 \\
\hline
\end{tabular}


The two independent measurements at each concentration give values that are in good agreement with each other. They are also in good agreement with the surface tensionGibbs equation results from our own surface tension data in Table 1. This immediately confirms that the Gibbs prefactor is one, i.e. that all three surfactants at natural pH are fully dissociated in the bulk solution despite the substantial increase in $\mathrm{pK}_{a}$ expected for the $\mathrm{C}_{8}$ spacer. ${ }^{7}$ The interesting straight line behaviour of the surface tension of the compound with the long spacer, which indicates constant surface excess over a large concentration range, is also replicated by the constant surface excess in the neutron data. Thus, this compound reaches its plateau adsorption at $1 / 10$ th of the CMC. This is highly unusual and we are not aware of a similar case. Taking our data and those of Chevalier et al. indicates that this is a definite feature of the compounds with the long spacer. In papers discussing the application of the Gibbs equation the evidence seems in general to indicate that nonionic surfactants reach a plateau before but close to the CMC, whereas ionic species do not, ${ }^{17,18}$ although there is no thermodynamic reason why a plateau of adsorption should be associated with the CMC. A plateau well below the CMC suggests that the micelle is relatively unstable compared with the saturated monolayer. This could be a result of a difficulty in accomodating a long spacer chain in a micelle and we discuss this further below in conjunction with the layer structure.

The structures of the saturated layers of the three betaines were determined at $3 \times C M C$ using the chain deuterated and spacer deuterated surfactants (not for the $\mathrm{C}_{1}$ spacer) in NRW and $\mathrm{D}_{2} \mathrm{O}$ and the fully protiated surfactant in $\mathrm{D}_{2} \mathrm{O}$. The five different isotopic reflectivity profiles were analysed using an extension of the kinematic model. Although the basis of the kinematic model has been fully described and applied elsewhere, ${ }^{15,16,31}$ for clarity we reproduce the main equations here. The betaines can be divided into two fragments, alkyl chain and the betaine group, which includes the spacer chain and the two charged groups. In the kinematic approximation, the reflectivity is then given by

$$
R=\frac{16 \pi^{2}}{\kappa^{2}}\left[b_{c}^{2} h_{c c}+b_{s}^{2} h_{s s}+b_{w}^{2} h_{w w}+2 b_{c} b_{s} h_{c s}+2 b_{c} b_{w} h_{c w}+2 b_{s} b_{w} h_{s w}\right]
$$


where the subscripts $c, s$ and $w$ denote alkyl chain, spacer group and water respectively, and $h_{i i}$ are the self- and $h_{i j}$ the cross-partial structure factors defined as

$$
\begin{aligned}
h_{i i} & =\operatorname{Re}\left(\hat{n}_{i}(\kappa) \hat{n}_{i}(\kappa)\right)=\left|\hat{n}_{i}(\kappa)\right|^{2} \\
h_{i j} & =\operatorname{Re}\left(\hat{n}_{i}(\kappa) \hat{n}_{j}(\kappa)\right)
\end{aligned}
$$

where $\hat{n}_{i}(\kappa)$ is the one dimensional Fourier transform of $n_{i}(z)$. The distributions of the surfactant fragments along the surface normal can be represented by Gaussian distributions,

$$
n_{i}(z)=n_{i 0} \exp \left(-\frac{4 z^{2}}{\sigma_{i}^{2}}\right)
$$

where $n_{i 0}$ is proportional to the surface density, $\sigma_{i}$ is the width parameter (full width at (height)/e) and $z$ is the distance along the surface normal. The self-partial structure factors, $h_{i i}$ are then

$$
h_{i i}=\frac{\pi \sigma_{i} n_{i 0}^{2}}{4} \exp \left(\frac{\kappa^{2} \sigma_{i}^{2}}{8}\right)
$$

For the Gaussian distributions of the alkyl chain and alkyl spacer the cross-partial structure factor, $h_{i j}$, has the simple form

$$
h_{i j}= \pm\left(h_{i i} h_{j j}\right)^{\frac{1}{2}} \cos \left(\kappa \delta_{i j}\right)
$$

where $\delta_{i j}$ is the separation of the centres of the two distributions along the surface normal direction. The $\sigma$ parameters are determined by a mixture of the intrinsic thickness of a fragment layer and the thermal fluctuations and, since these may sometimes be comparable, it can be difficult to extract useful structural information from them. However, the value of $\delta$ is determined by correlations between the fragments in each molecule and these are largely determined by the intra-layer structure and generally give rise to the main interference effects in the reflectivity. The distribution of water is more complicated and, following a previous model, is taken to be determined by space filling up to a certain distance into the surfactant 
layer followed by a decay to zero,

$$
\phi_{w}(z)=1-\phi_{c}-\phi_{s} ; z \leq \xi
$$

where $\phi$ are volume fractions and $\xi$ is a cut-off distance, ${ }^{32}$ and

$$
\phi_{w}(z)=\phi_{w}(\xi) \exp \left[-\frac{(z-\xi)^{2}}{\sigma^{2}}\right] ; z \geq \xi
$$

Thus, there is no empty space below the cut-off distance. Above this the water decays following a half-Gaussian. This distribution can be thought of as defining the region of water over which the capillary waves exert an effect. The Fourier transform of this has to be calculated numerically but again the important parameters for assessing the structure are the equivalent $\delta_{c w}$ or $\delta_{s w}$ parameters determined by the correlations between the water and the surfactant fragments. The procedure is to use a set of trial parameters and to compute $R$ and optimize a simultaneous fit to the full set of reflectivity profiles, basically to determine the individual structure factors, especially the cross terms in Eqn (4). For this analysis, the number of independent parameters for the betaines is 7 , and these are the surface excess of surfactant, the widths of the alkyl chain and betaine fragments, the separations of betaine from alkyl chain and from water, and the cut-off for the water and decay length for the water profile. For a tighter comparison of these three similar surfactants we now introduce a different parameterization within the framework of the equations above.

We have shown elsewhere that the width of the distribution of a surfactant fragment is determined in part by the length of the projection of the fragment along the normal direction, denoted $l$, and in part by the thermal roughness, $w$, from the capillary waves such that the parameter $\sigma$ in Eqn (6) is given by ${ }^{15,33}$

$$
\sigma^{2}=l^{2}+w^{2}
$$


Since the capillary waves affect the whole layer, $w$ should be the same for all the fragments, including the water decay in Eqn (10). In addition, since the two fragments of the surfactant are attached the distance between their centres must be constrained to be

$$
\delta_{c s}=\frac{1}{2}\left(l_{s} \pm l_{c}\right)
$$

where the \pm depends on whether the angle between the two projections along the surface normal is greater or less than $90^{\circ}$. There are now only 6 independent parameters, which are the surface excess, the two fragment projections along the surface normal, the thermal roughness, the water cut-off, and either the chain-water separation or the spacer-water separation. Furthermore, since the thermal roughness $w$ is expected to be mainly determined by the surface tension, and the surface tensions are not very different for the three betaine surfactants, it is reasonable to make the approximation that $w$ is the same for all three, which further reduces the number of parameters for the set of surfactants. The fits to the reflectivity profiles and the corresponding fragment distributions based on this model are plotted in Figures 3 and 4 and the parameters are given in Table 3. The results in Figure 4 are plotted as volume fraction of the various fragments, obtained using the scattering lengths in Table 1 of the Supporting Information. The fits should be judged as a set in that the unusually tight constraints put on the fitting model lead to some fits that could be improved either by relaxing the constant $w$ restriction or allowing the independent variation of $\delta_{c s}$. Thus, the fits for the samples with only the deuterated spacer (the lowest ones in Figure 3) would be better fitted with a greater thermal contribution. However, as outlined above the important parameter is the separation and the constraints on the fitted parameters highlight the differences in the structural correlations between the fragments for the three surfactants. 


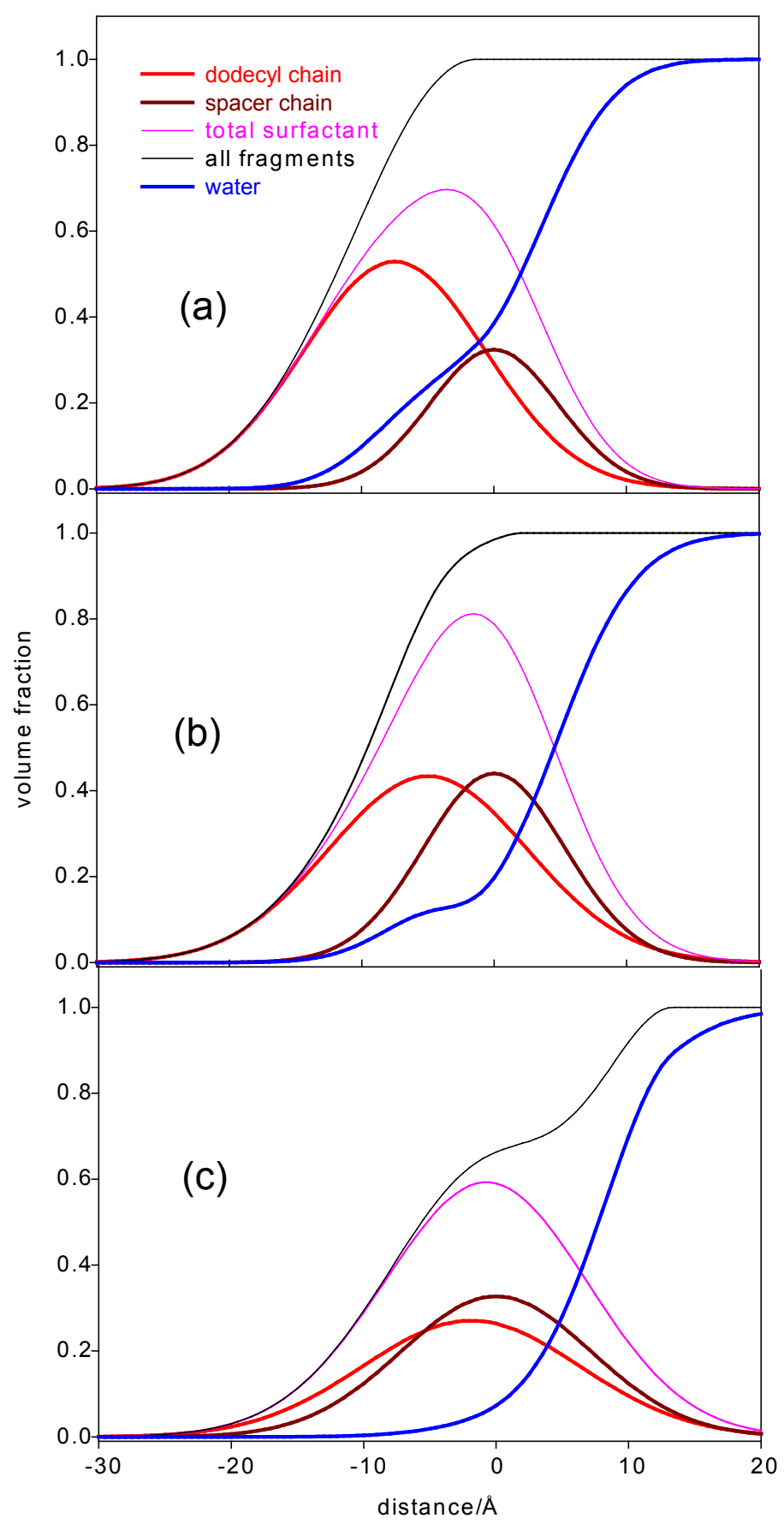

Figure 4: Distributions of the different fragments in layers of carboxybetaines at the air-water surface that best fit the reflectivity data of Figure 3. The spacer consists of the quaternary ammonium group and carboxy- groups separated by (a) 1, (b) 4 and (c) 8 methylene groups. The centre of the spacer distribution was fixed at zero for each system for convenience. 
Table 3: Structural parameters derived from fitting neutron reflection data from three betaines at $3 \times \mathrm{CMC}$ using five different contrasts with NRW and $\mathrm{D}_{2} \mathrm{O}$. The symbols are explained in the text. ${ }^{a}$ depends directly on $l_{c}$ and $l_{s} .{ }^{b}$ The parameter defining the cut-off of the water space filling region is indirectly incorporated into $\delta_{s w}$.

\begin{tabular}{|c|cccccc|}
\hline compound $(c)$ & $l_{c} \pm 3 \AA$ & $l_{s} \pm 3 \AA$ & ${ }^{a} \delta_{c s}$ & ${ }^{b} \delta_{s w} \pm 1 \AA$ & $w \pm 3 \AA$ & $A \pm 3 \AA^{2}$ \\
\hline $\mathrm{C}_{12} \mathrm{C}_{1}$ betaine & 11 & 1 & -6 & -0.5 & 11 & 48 \\
$\mathrm{C}_{12} \mathrm{C}_{4}$ betaine & 13 & 5 & -4 & -3 & 11 & 52 \\
$\mathrm{C}_{12} \mathrm{C}_{8}$ betaine & 15 & 12 & -1.5 & -6 & 11 & 80 \\
\hline
\end{tabular}

There are two trends that are easily identified. The first is that the projection of the $\mathrm{C}_{12}$ chain along the surface normal increases with the betaine spacer length. The length as defined by the $1 / e$ width of a Gaussian is about 0.9 of the true length ${ }^{15}$ and the extended length of the $\mathrm{C}_{12}$ chain is $16 \AA$. Thus, in the presence of the $\mathrm{C}_{1}$ spacer the projection of the $\mathrm{C}_{12}$ chain and its known length leads to a tilt of about $30^{\circ}$ from the vertical but this becomes more or less vertical in the presence of the $\mathrm{C}_{8}$ spacer. The second trend is that the spacer chain of the betaine group changes orientation even more dramatically. For the $\mathrm{C}_{1}$ compound the intrinsically short length of the spacer means its thickness is close to the thermal roughness. For the $\mathrm{C}_{8}$ compound the fully extended length is estimated to be about $15 \AA$ (10 $\AA$ from the $\mathrm{C}_{8} \mathrm{H}_{16}$ and $5 \AA$ from the two charged groups). Thus the tilt of the spacer as as a whole is about $30^{\circ}$ from the vertical, almost exactly the same as the dodecyl chain in the $\mathrm{C}_{1}$ surfactant. This is a surprising result because it shows that the carboxyl group must be completely out of the water, rather than looped as suggested by Chevalier et al. and we will discuss this further below. Note that because of the way we have combined the projections of alkyl chain and spacer the $\delta_{c s}$ is required to be consistent with the description above and therefore adds no new information.

A different and more subtle measure of the nature of the surface layer is the overlap of each unit with water, i.e. the integral of the product of the distributions of the unit and water along the surface normal. A small overlap between a hydrophobic unit and water suggests that the hydrophobic unit is more effectively screening the high energy surface of 
the water than if there is a large overlap. It is easy to use the distributions of Figure 3 to calculate and normalize these overlaps so that they are directly comparable between the three systems. The calculated overlaps are 3.8, 3.3 and 3.0 between the dodecyl chain and water and 8.1, 5.3 and 3.7 between spacer and water for the $\mathrm{C}_{1}, \mathrm{C}_{4}$ and $\mathrm{C}_{8}$ spacers respectively, where the values are normalized both to the volume fraction per dodecyl chain and to the area per molecule. These numbers show that within error the apparent effectiveness of the dodecyl chain is more or less the same across the series. However, the $\mathrm{C}_{1}$ and $\mathrm{C}_{4}$ spacers overlap strongly with water and the methylene groups of these spacers must be significantly immersed. This does not matter much for $\mathrm{C}_{12} \mathrm{C}_{1}$ betaine but it leads to a significant loss of surface energy for $\mathrm{C}_{12} \mathrm{C}_{4}$ betaine leading to a larger $\gamma_{C M C}$, as seen in Figure 2. However, the low value of the overlap for the $\mathrm{C}_{8}$ spacer shows that it is as effective at screening the water per unit volume fraction as the dodecyl group. The overall screening of water per unit area of surfactant is therefore approximately the same for $\mathrm{C}_{12} \mathrm{C}_{1}$ betaine and $\mathrm{C}_{12} \mathrm{C}_{8}$ betaine. However, the contribution from the spacer group will be partially offset by the exposed polar carboxyl at the surface, which will have a higher surface energy than a methyl group, and overall the $\gamma_{C M C}$ value could then be a bit higher for the $\mathrm{C}_{8}$ compound, as observed.

That the $\mathrm{C}_{8}$ spacer is well extended and has an overlap with water that is approximately the same as the dodecyl chain, shows that the carboxyl group must be on average more or less completely out of the water. An ionized carboxyl group would be unstable in the low dielectric environment of the outer part of the layer and combined with its much higher $\mathrm{pK}_{a}$ this raises the possibility that $\mathrm{C}_{12} \mathrm{C}_{8}$ betaine is adsorbed in its undissociated carboxylic acid form. The conventional argument against the presence of what would then be the simple cationic form of the betaine at the surface is that the prefactor in the Gibbs equation would be 2. Wustneck et al. ${ }^{6}$ have indeed shown that the adsorption of the salt of $\mathrm{C}_{12} \mathrm{C}_{1}$ betaine, i.e. the salt with $\mathrm{HBr}$ of $\mathrm{HCl}$ does follow the Gibbs equation with a prefactor of 2. However, the conventional argument for a prefactor of 2 is not appropriate unless the zwitterion is in salt form. The equilibrium between bulk and surface can be equivalently expressed either 
as (see Guggenheim ${ }^{34}$ for the treatment of the independence of components in the Gibbs equation)

$$
{ }^{-} \mathrm{OOCC}_{8} \mathrm{D}_{16} \mathrm{NHMe}_{2}^{+}(\text {bulk }) \leftrightarrows-\mathrm{OOCC}_{8} \mathrm{D}_{16} \mathrm{NHMe}_{2}^{+} \text {(surface) }
$$

or

$$
{ }^{-} \mathrm{OOCC}_{8} \mathrm{D}_{16} \mathrm{NHMe}_{2}^{+}(\text {bulk })+\mathrm{H}_{2} \mathrm{O} \leftrightarrows \mathrm{HOOCC}_{8} \mathrm{D}_{16} \mathrm{NHMe}_{2}^{+}(\text {surface })+\mathrm{OH}^{-}
$$

The first case is what is conventionally assumed and has a Gibbs prefactor of 1 . However, the second case also has a Gibbs prefactor of 1 because the concentration of $\mathrm{OH}^{-}$ion in the bulk is constant at natural pH. Neither the adsorbed amount nor the degree of hydrolysis (formation of $\mathrm{COOH}$ ) is sufficient to affect the overall $\mathrm{pH}$. Thus the Gibbs equation argument shows only that the $\mathrm{C}_{12} \mathrm{C}_{8}$ betaine is fully dissociated in the bulk solution. It does not help decide whether or not it is dissociated in the surface layer.

The neutron reflection experiment therefore shows that the orientation of the spacer-head group in adsorbed layers of carboxybetaines with long spacers leads to the carboxyl groups being approximately aligned in a higher plane of the surface than the cationic quaternary ammonium groups. If, as argued above, the carboxyl groups lose their charge in the layer (as drawn in the TOC graphic), there will be a significant reduction in the lateral electrostatic repulsion, especially because there is no high dielectric constant from water at the level of the carboxylic groups. It is interesting to note that, by the more direct method of FTIR, Gershevitz and Sukenik have shown that carboxyl terminated self-assembled monolayers are also mostly nonionized, although this seems to be more of a proximity effect. ${ }^{35}$ For micellization, such an effect can only occur if the betaine is incompletely dissociated in the bulk solution and the Gibbs analysis indicates that it is not. The situation cannot be different in the micelle because the curvature at the surface of the micelle will tend to force the spacer groups to point outwards from the micelle and the high dielectric environment will ensure that the carboxylate group remains fully ionized provided $\mathrm{pK}_{a}$ is not too high (the phase separation observed for the $\mathrm{C}_{10}$ spacer in the phosphocholines by Chevalier et al. ${ }^{36}$ suggests 
that this point may have been reached in those systems). This difference means that the formation of a saturated monolayer at the air-water interface is unusually strongly favoured in comparison with micellization. We believe that this may be the origin of the unusually low concentration, relative to the CMC, at which the plateau of adsorption is reached and hence the long straight line section observed in the $\gamma-\ln c$ plot. In the more complete series studied by Chevalier et al. the straight line region starts to appear from about the $\mathrm{C}_{4}$ spacer, i.e. about the same point as the maximum in the CMC and $\gamma_{C M C}$. This link is consistent with the mechanism suggested by the neutron reflection results.

\section{Acknowledgements}

We thank STFC for the provision of neutron beam time at the ISIS facility. KM thanks the China Scholarship Council for the award of a scholarship.

\section{References}

(1) Hodge, D. J.; Laughlin, R. G.; Ottewill, R. H.; Rennie, A. R. Micellization of Ultralong Chain Surfactant. Langmuir 1991, 7, 878-884.

(2) Welling-Wester, S.; Feijlbrief, M.; Koedijk, D. G. A. M.; Welling, G. W. Detergent extraction of Herpes Simplex Virus type 1 Glycoprotein D by Zwitterionic and Non-ionic Detergents and Purification by Ion-exchange High-performance Liquid Chromatography. J. Chromatography A 1998, 816, 29-37.

(3) Rhein, L. D.; Schlossman, M.; O'Lenick, A.; Somasundaran, P. Surfactants in Personal Care Products and Decorative Cosmetics, 3rd ed.; CRC Press: Baton Rouge, U.S.A., 2006.

(4) Robinson, R. A.; Stokes, R. H. Electrolyte Solutions, 2nd ed.; Butterworth Scientific Publications, 1959; pp 346-350. 
(5) Laughlin, R. G. Fundamentals of the Zwitterionic Hydrophilic Group. Langmuir 1991, ๆ, 842-847.

(6) Wustneck, R.; Kriwanek, T.; Herbst, M.; Wasow, G.; Haage, K. The adsorption behaviour of long-chain N-alkyl betaines and their hydrobromides. Colloids Surfaces 1992, $66,1-9$.

(7) Weers, J. G.; Rathman, J. F.; Axe, F. U.; Crichlow, C. A.; Lafayette, D. F.; ; Scheuing, D. R.; Wiersema, R. J.; Zielske, A. G. Effect of the Intramolecular Charge Separation Distance on the Solution Properties of Betaines and Sulfobetaines. Langmuir 1991, 7, 854-867.

(8) Gane, R.; Ingold, C. K. Electrometric Titration Curves of Dibasic Acids. Part I. Normul Acids. J. Chem. Soc. 1928, 1594-1600.

(9) Gane, R.; Ingold, C. K. Electrometric Titration Curves of Dibasic Acids. Part IV. Corrections for Interionic Effects and for Solvent Electrostriction. J. Chem. Soc. 1931, 2153-2169.

(10) Kirkwood, J. G.; Westheimer, F. H. The Electrostatic Influence of Substituents on the Dissociation Constants of Organic Acids. I. J. Chem. Phys. 1938, 6, 506-512.

(11) Schwarzenbach, G. Electrostatic and non-electrostatic contributions to ion association in solution. Pure Applied Chem. 1970, 24, 307-334.

(12) Kornyshev, A. A.; Vorotyntsev, M. A.; Nielsen, H.; Ulstrup, J. Non-local Screening Effects in the Long Range Interionic interaction in a Polar Solvent. Journal of the Chemical Society Faraday Transactions 1982, 78, 217-241.

(13) Chevalier, Y.; Germanaud, L.; Le Perchec, P. Micellar properties of zwitterionic phosphobetaine amphiphiles in aqueous solution: Influence of the intercharge distance. Colloid Polymer Sci. 1988, 266, 441-448. 
(14) Chevalier, Y.; Storet, Y.; Pourchet, S.; Le Perchec, P. Tensioactive Properties of Zwitterionic Carboxybetaine Amphiphiles. Langmuir 1991, 7, 848-853.

(15) Lu, J. R.; Li, Z. X.; Smallwood, J.; Thomas, R. K.; Penfold, J. Detailed Structure of the Hydrocarbon Chain in a Surfactant Monolayer at the Air-water Interface: Neutron Reflection from Hexadecyltrimethylammonium Bromide. J. Phys. Chem. 1995, 99, 8233-8243.

(16) Lu, J. R.; Thomas, R. K.; Penfold, J. Surfactant layers at the air/water interface: structure and composition. Adv. Colloid Interface Sci. 2000, 84, 143-304.

(17) Li, P. X.; Li, Z. X.; Shen, H. H.; Thomas, R. K.; Penfold, J.; Lu, J. R. Application of the Gibbs Equation to the Adsorption of Nonionic Surfactants and Polymers at the Air/Water Interface: Comparison with Surface Excesses Determined Directly using Neutron Reflectivity. Langmuir 2013, 29, 9324-9334.

(18) Xu, H.; Li, P. X.; Ma, K.; Thomas, R. K.; Penfold, J.; Lu, J. R. Limitations in the Application of the Gibbs Equation to Anionic Surfactants at the Air/Water Surface: Sodium Dodecylsulfate and Sodium Dodecylmonooxyethylenesulfate Above and Below the CMC. Langmuir 2013, 29, 9335-9351.

(19) Albu, S.; Sverko, E.; Arts, M. T.; Capretta, A. Synthesis of 5 (Z), 11 (Z)-eicosadienoic acid as a biomarker for trophic transfer. Tetrahedron Letters 2011, 52, 787-788.

(20) Nguyen, J. T.; McEwen, C.; Knaus, E. E. Hantzsch 1, 4-dihydropyridines containing a nitrooxyalkyl ester moiety to study calcium channel antagonist structure-activity relationships and nitric oxide release. Drug Development research 2000, 51, 233-243.

(21) Baldwin, S. W.; Wilson, J. D.; Aube, J. Regiochemistry in the intramolecular cycloadditions of substituted 5-alkenyl and 6-alkenyl nitrones. J. Org. Chem. 1985, 50, 4432-4439. 
(22) Maiti, G.; Roy, S. C. A mild and efficient method for selective deprotection of tetrahydropyranyl ethers to alcohols. J. Org. Chem. 1996, 61, 6038-6039.

(23) Khlebnikov, A. F.; Kozhushkov, S. I.; Yufit, D. S.; Schill, H.; Reggelin, M.; Spohr, V.; de Meijere, A. A Novel Type of Chiral Triangulane-Based Diphosphane Ligands for Transition Metals. Eur. J. Org. Chem. 2012, 8, 1530-1545.

(24) Buchi, G.; Jeger, O. ber einige Verbindungen mit dem Kohlenstoffgerst des Bicyclo[0,5,5]-dodecans. Helvetica Chim. Acta 1949, 32, 538-544.

(25) Kurata, T.; Kurita, N. Synthesis of 17 membered-ring oxalactones and its odor characteristics. Fain Kemikaru 2008, 37, 43-49.

(26) Bunce, N. J. Mercury salt pathway for the degradation of carboxylic acids to alkyl halides using halogen and mercuric oxide. J. Org. Chem. 1972, 37, 664-669.

(27) Webster, J. R. P.; Langridge, S.; Dalgliesh, R. M.; Charlton, T. R. Reflectometry techniques on the Second Target Station at ISIS: Methods and science. Eur. Phys. J. Plus 2011, 126, 112.

(28) Jackson, A. J.; Li, P. X.; Dong, C. C.; Thomas, R. K.; Penfold, J. Structure of Partially Fluorinated Surfactant Monolayers at the Air-Water Interface. Langmuir 2009, 25, $3957-3965$.

(29) Born, M.; Wolf, E. Principles of Optics, 7th ed.; Cambridge University Press, 2003; Chapter 2.

(30) Hines, J. D.; Garrett, P. R.; Rennie, G. K.; Thomas, R. K.; Penfold, J. Structure of an Adsorbed Layer of n-Dodecyl-N,N-dimethylamino Acetate at the Air-Solution Interface As Determined by Neutron Reflection. J. Phys. Chem. 1997, 101, 7121-7126.

(31) Crowley, T. L.; Lee, E. M.; Simister, E. A.; Thomas, R. K. The use of contrast variation in the specular reflection of neutrons from interfaces. Physica B 1991, 173, 143-156. 
(32) Penfold, J.; Thomas, R. K. Solvent distribution in non-ionic surfactant monolayers. Phys. Chem. Chem. Phys. 2002, 4, 2648-2652.

(33) Pleshanov, N. K.; Pusenkov, V. M.; Schebetov, A. F.; Peskov, B. G.; Shmelez, G. E.; Siber, E. V.; Soroko, Z. N. On the use of specular neutron reflection in the study of roughness and interdiffusion in thin-film structures. Physica B 1994, 198, 27-32.

(34) Guggenheim, E. A. Thermodynamics, 5th ed.; North Holland Publishing Company: Amsterdam, 1967; pp 293-297.

(35) Gershevitz, O.; Sukenik, C. N. In Situ FTIR-ATR Analysis and Titration of Carboxylic Acid-Terminated SAMs. J. Am. Chem. Soc. 2004, 126, 482-482.

(36) Chevalier, Y.; Melis, F.; Dalbiez, J. Structure of Zwitterionic Surfactant Micelles: Micellar Size and Intermicellar Interactions. J. Phys. Chem. 1992, 96, 8614-8619.

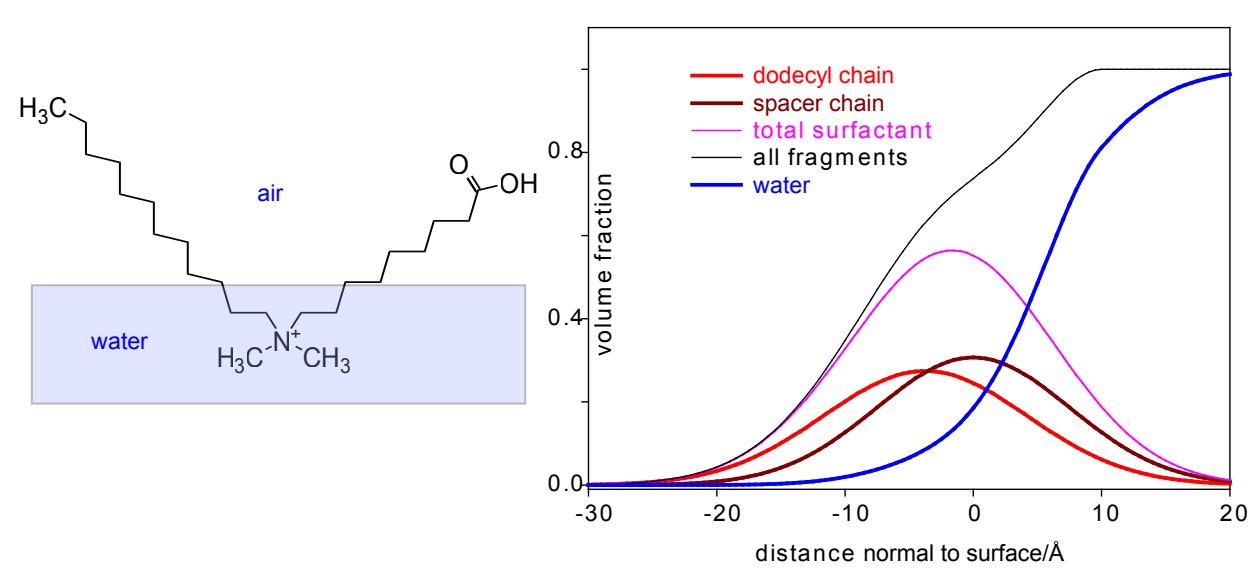

